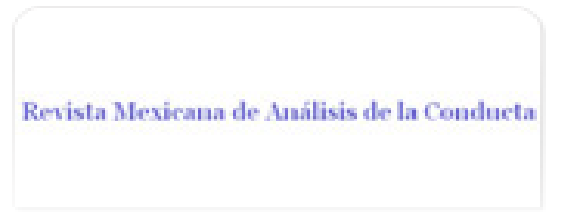

Revista Mexicana de Análisis de la Conducta ISSN: 0185-4534

editora@rmac-mx.org

Sociedad Mexicana de Análisis de la Conducta México

Ortiz-Rueda, Gerardo; Cruz-Alaniz, Yuria

EL PAPEL DE LA PRECISIÓN INSTRUCCIONAL Y LA RETROALIMENTACIÓN EN LA EJECUCIÓN Y DESCRIPCIONES POSCONTACTO

Revista Mexicana de Análisis de la Conducta, vol. 37, núm. 1, abril-julio, 2011, pp. 69-87

Sociedad Mexicana de Análisis de la Conducta

Guadalajara, México

Disponible en: http://www.redalyc.org/articulo.oa?id=59319102006

Cómo citar el artículo

- Número completo

- Más información del artículo

Página de la revista en redalyc.org

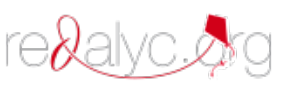

Sistema de Información Científica

Red de Revistas Científicas de América Latina, el Caribe, España y Portugal Proyecto académico sin fines de lucro, desarrollado bajo la iniciativa de acceso abierto 


\title{
EL PAPEL DE LA PRECISIÓN INSTRUCCIONAL Y LA RETROALIMENTACIÓN EN LA EJECUCIÓN Y DESCRIPCIONES POSCONTACTO
}

\author{
INSTRUCTIONAL CONTROL AND FEEDBACK EFFECTS ON \\ PERFORMANCE AND POSCONTACT DESCRIPTIONS
}

\author{
GERARDO ORTIZ-RUEDA ${ }^{1}$ Y YURIA CRUZ-ALANIZ ${ }^{2}$ \\ 1 Centro de Estudios e Investigaciones en Comportamiento \\ Universidad de Guadalajara, México \\ 2 Posgrado en Ciencias del Comportamiento \\ Universidad de Guadalajara, MÉxICO
}

\begin{abstract}
Resumen
En el siguiente estudio se exploraron los efectos de las descripciones precontacto y la frecuencia de retroalimentación sobre la ejecución y descripciones poscontacto en tareas de igualación de la muestra de primer orden. Participaron 36 estudiantes agrupados en 6 grupos, los cuales se diferenciaban por el tipo de descripción precontacto (i.e. manipulando el componente Respuesta). La precisión de las descripciones precontacto se formuló de acuerdo a la taxonomía propuesta por Ortiz, González y Rosas (2008). Los resultados muestran que los sujetos que recibieron una descripción precontacto Específica Pertinente junto con una densidad de retroalimentación Acumulada, realizan las mejores ejecuciones y descripciones poscontacto. Los resultados sugieren que el componente Respuesta (R) es aquél con mayor importancia para la adquisición de una función instruccional de las descripciones precontacto.
\end{abstract}

Palabras clave: Instrucciones, reglas, retroalimentación, descripciones precontacto, descripciones poscontacto.

El primer autor agradece el apoyo recibido por parte del Consejo Nacional de Ciencia y Tecnología (Beca 206677) en la realización de esta investigación. El presente estudio fue conducido como parte de las actividades en el posgrado del segundo autor bajo la dirección del primer autor. Los autores contribuyeron en la misma proporción en la realización del presente estudio y agradecen los valiosos comentarios de tres revisores anónimos que permitieron mejorar el manuscrito. Dirigir toda correspondencia a los autores a: Centro de Estudios e Investigaciones en Comportamiento, calle Francisco de Quevedo 180, Col. Arcos Vallarta. Guadalajara, Jalisco, México, C.P. 44130. Correos electrónicos oruga@cencar.udg.mx y yuria20@hotmail.com 
Abstract

In the present study we explored the effects of pre-contact descriptions (i.e. instructions) precision (i.e. specific and pertinent, generic and pertinent, specific and non pertinent, generic and non pertinent, irrelevant, absent) as well as the feedback frequency (i.e. continuous, accumulated) on instrumental performance and post-contact descriptions (i.e. rules) on a first order matching-to-sampling task. The precision of the pre-contact description given to subjects, as well as the analysis of post-contact descriptions made by all participants, was qualified according to the proposal made for Ortiz, González \& Rosas (2008). 36 students were assigned to one of six experimental groups. Results show that subjects which received a Specific and Pertinent (SP) description with an accumulated feedback frequency had the best instrumental performance and elaborated the best post-contact descriptions. Obtained data suggest the relevance of the Response component $(R)$ in the acquisition of an instructional function by a pre-contact description.

Key words: Instructions, rules, feedback, precontact descriptions, poscontact descriptions.

Desde el momento en el que Skinner (1966) propone la utilización del término "conducta gobernada por reglas" como una fuente alternativa a las contingencias de control sobre la conducta, en el Análisis Experimental de la Conducta se ha discutido una problemática relacionada con la existencia, tipos y función de dicha fuente y fenómenos asociados. Como parte de esa discusión, surge el área que se ha denominado control instruccional, donde se asume la existencia independiente de reglas e instrucciones (e.g. Baron \& Galizio, 1983; Cerutti, 1989).

Diversos autores han referido que tanto reglas como instrucciones son descripciones verbales que describen la ejecución o contingencias que enfrentará o enfrentó un individuo (e.g. Ortiz et al., 2006; Ribes \& Martínez, 1990; Ribes, Moreno \& Martínez, 1995; Ribes, Torres \& Ramírez, 1996). No obstante, aún cuando se han mencionado diversas diferencias entre ambos conceptos (e.g. Chase \& Danforth, 1991; Hayes, 1989; Harzem, Lowe \& Bagshaw, 1978; Peláez \& Moreno, 1998; Ribes, 2000; Rosenfarb, Newland, Brannon \& Howey, 1992; Trigo, Martínez \& Moreno, 1995), todavía no es posible unificar la terminología y, en muchas ocasiones, suelen utilizarse como sinónimos (e.g. Hayes, Brownstein, Haas \& Greenway, 1986), posiblemente porque no se trata de términos técnicos específicos (e.g. Ortiz, 2005; Ribes, 2000)

Una posibilidad que plantean Ortiz (2005), Ortiz et al. (2006) y Ortiz, González \& Rosas (2008) es la utilización de los términos descripciones pre y poscontacto para hacer referencia a instrucciones y reglas. Dicha posibilidad parte del supuesto de que el aspecto más importante a tomar en cuenta para diferenciarlos no estriba en la forma que pueden adquirir, sino en el momento en que se presentan 
y la función que cumplen. Así, una descripción precontacto contingencial puede adquirir funciones instruccionales (i.e. restringir el rango de respuesta de un sujeto próximo a enfrentarse a las contingencias), mientras que una descripción poscontacto contingencial puede adquirir una función de regla, a partir de una abstracción de las contingencias enfrentadas que se suele realizar para facilitar el enfrentamiento de situaciones posteriores semejantes (e.g. Ribes, 2000).

Ortiz et al. (2006) señalan que "... las variables relevantes para que una descripción funcione como instrucción (y se diferencie de una regla) parecen implicar su precisión respecto a la contingencia que describe, a partir de una exitosa ejecución de la tarea, así como la posibilidad de ser utilizada como instrucción en un momento distinto al de su realización." (p. 105).

Con el fin de estudiar la función instruccional de las descripciones precontacto y las variables que permiten a una descripción de éste tipo adquirir dicha función, se han realizado diversas investigaciones manipulando aspectos tales como la correspondencia entre la descripción presentada y las consecuencias (e.g. Buskist \& Miller, 1986; Galizio, 1979; Ribes \& Martínez,1990) y el efecto de la retroalimentación (e.g. Baron, Kaufmann \& Stauber 1969; Guerrero-Radillo \& Ortiz, 2007; Martínez, Ortiz \& González, 2002).

Otro aspecto que ha sido de interés se relaciona con la precisión de las descripciones precontacto (e.g. Hayes et al., 1986; Martínez, Ortiz \& González, 2007; Ortiz \& González, 2010a). Dicha variable (i.e. precisión de descripciones) es posible analizarla utilizando la taxonomía propuesta por Ortiz, González \& Rosas (2008), que sugieren que las descripciones pueden estar integradas por tres componentes: 1) Situación de Estímulo (SE), 2) Componente Respuesta (R) y, 3) el Componente Consecuencia (C). El componente SE puede variar, por ejemplo, en la distribución de componentes físicos y el tipo, la cantidad o la locación de los estímulos; el componente $\mathrm{R}$ en el criterio de ajuste de la tarea y la acción que se requiere para realizar la tarea, mientras que el componente $C$ en la presencia, frecuencia y modalidad de presentación de la retroalimentación. Tomando en cuenta la posibilidad de que los elementos de cada componente tengan distintas cualidades (i.e. presencia, relevancia, especificidad y precisión), la taxonomía identifica seis tipos posibles de descripciones, a saber: 1) Específico Pertinente, 2) Específico No Pertinente, 3) Genérico Pertinente, 4) Genérico No Pertinente, 5) Irrelevante, 6) Ausente.

En diversas investigaciones realizadas con el fin de estudiar la posible diferencia funcional entre instrucciones y reglas, utilizando la taxonomía antes expuesta, se ha encontrado que las descripciones precontacto (i.e. que adquieren función instruccional) con mejor precisión respecto a las contingencias que describen, propician una más rápida adquisición y un mejor mantenimiento de una conducta (e.g. Guerrero-Radillo \& Ortiz, 2007; Ortiz, de la Rosa, Padilla, Pulido \& Vélez, 2008; Ortiz, González, Rosas \& Alcaraz, 2006; Ortiz \& González, 2010a; Ortiz, Pacheco, Bañuelos \& Plascencia, 2007). Tales resultados son consistentes con la 
literatura respecto al supuesto de que será mejor el seguimiento de instrucciones (i.e. control instruccional) en la medida en que la instrucción (i.e. descripción precontacto con función isntruccional) corresponda con la situación que describe y con las consecuencias obtenidas por seguirlas (e.g. Buskist \& Miller, 1986; Catania, Shimoff \& Matthews 1989; DeGrandpre \& Buskist, 1991; Lefrancois, Chase \& Joyce, 1988; Martínez, Ortiz \& González, 2002; Martínez \& Ribes, 1996; Newman, Hemmes, Buffington \& Andreopoulus, 1991; Ribes \& Rodríguez, 2001).

Por ejemplo, Ortiz et al. (2006) realizaron dos experimentos en los que evaluaron los efectos de la precisión instruccional (específica vs. genérica) y la frecuencia de retroalimentación (cero, continua o acumulada) tanto en el seguimiento de descripciones precontacto (i.e. función instruccional) como en la elaboración de descripciones poscontacto contingencial (i.e. función de regla). En el Experimento 1 , participaron 30 estudiantes universitarios que fueron asignados aleatoriamente a uno de seis grupos experimentales que se diferenciaron por el tipo de descripción precontacto recibida en la segunda fase del estudio, así como por la frecuencia de la retroalimentación que recibían respecto de su ejecución; en ninguno de los casos los participante sabían cuál era la finalidad de realizar la descripción poscontacto (i.e. ser utilizada como descripción precontacto para otros participantes). Los resultados mostraron que la precisión de la descripción precontacto y la frecuencia de la retroalimentación tienen efectos diferenciales en la ejecución instrumental del sujeto (i.e. ejecuciones superiores con descripciones específicas o con retroalimentación continua), así como en falta de correspondencia entre los niveles de dicha ejecución instrumental y el tipo de descripción poscontacto realizada.

En el segundo experimento, investigaron el uso de descripciones realizadas por diferentes sujetos del primer experimento como descripciones precontacto para sujetos de los grupos correspondientes a este estudio. En general se observaron ejecuciones instrumentales que oscilaron alrededor de los 12 de los 36 aciertos posibles, ejecución que podría considerarse al azar, así como una mayor muestra de descripciones de tipo irrelevante respecto del experimento previo. A la luz de estos resultados, Ortiz et al. (2006) sugirieron que el componente R parece jugar un papel preponderante para que una descripción adquiera una función instruccional facilitando el que la ejecución de los sujetos sea sistemática, ya que en las descripciones precontacto que se otorgó a los sujetos en el segundo experimento, generalmente el componente mencionado se podía calificar cuando mucho como de tipo genérico pertinente, haciéndole falta uno o varios de los elementos que lo integran (i.e. maniobra de respuesta, acción requerida, criterio de respuesta).

Ortiz (2010) realizó una replicación sistemática de estos estudios modificando el conocimiento que tenían los participantes del primer estudio respecto del uso que se le daría a la descripción poscontacto que realizarían (i.e. ser utilizada como descripciones precontacto por los participantes del estudio 2). En general, los resultados son similares a los encontrados por Ortiz et al. (2006), encontrándo- 
se diferencias en cuanto el tipo de descripción poscontacto realizada por los participantes del Experimento 1 (i.e. mayor precisión del componente R), así como por el mayor seguimiento instruccional de los participantes del Experimento 2. Ortiz (2010) sugiere que los resultados parecen implicar la independencia entre las descripciones pre y poscontacto contingencial; mientras que la descripción realizada posterior a la exposición a las contingencias parece ser afectada por variables tales como la falta de pericia para realizarla, el tipo de protocolo utilizado para solicitarla y las indicaciones que se dan respecto de la realización de dicha tarea, al ser utilizada como instrucción su eficiencia parece depender de la interacción de variables tales como la correspondencia con las contingencias (i.e. situación de estímulo, respuesta criterio y consecuencias recibidas) y la historia de seguimiento instruccional que tiene el sujeto, entre otras.

En el mismo sentido, Ortiz \& González (2010b) sugieren que el componente R parece cumplir un papel fundamental, y que de los elementos que lo componen (i.e. maniobra por realizar, tipo de respuesta requerida y criterio de respuesta) es el elemento relacionado con el ajuste (i.e. criterio de respuesta) el que parece jugar un papel crítico para que una descripción precontacto desempeñe una función instruccional.

Bajo esta lógica, y con el fin de abonar al entendimiento de la manipulación de la precisión del componente $\mathrm{R}$ sobre la adquisición de una función instruccional por parte de la descripción precontacto, el objetivo del presente estudio fue explorar los efectos de la precisión de descripciones precontacto, manipulando el componente Respuesta, y la frecuencia de retroalimentación, sobre el seguimiento de dichas descripciones precontacto (i.e. adquisición de una función instruccional) y el tipo de descripciones postcontacto realizadas por los sujetos, en tareas de igualación de la muestra.

\section{Método}

Sujetos

Participaron voluntariamente 36 estudiantes universitarios (32 mujeres y 4 hombres) pertenecientes a la Universidad de Guadalajara, con un rango de edad de 18 a 35 años quienes fueron contactados a través del profesor de una de sus materias. Todos ellos carecían de experiencia en la tarea experimental.

Aparatos y Escenario

Todas las sesiones experimentales se llevaron a cabo en los cubículos del Laboratorio de Conducta Humana del Centro de Estudios e Investigaciones en Comportamiento de la Universidad de Guadalajara. Los cubículos contaban con iluminación artificial y tenían dimensiones aproximadas de $3 \mathrm{~m}$ de largo por $3 \mathrm{~m}$ de ancho.

Cada cubículo contaba con una computadora personal de marca comercial con procesador Pentium IV. La programación de los estímulos, la aplicación de la 
tarea experimental y la recolección de las respuestas de los sujetos se llevó a cabo mediante el programa Toolbook II Instructor.

\section{Tarea Experimental}

Se utilizó un procedimiento de igualación de la muestra de primer orden. Cada ensayo consistió en la presentación de un estímulo muestra (Em) colocado al centro de la pantalla y tres estímulos comparativos (ECo) alineados horizontalmente en la parte inferior de la pantalla. Cada arreglo de estímulos contenía, con respecto al Em, un ECo idéntico en forma y en color, otro semejante en forma o en color y otro diferente tanto en forma como en color. La tarea consistió en elegir de entre los tres ECós, aquel que guardara una relación de semejanza con el Em (color o forma).

Los colores de los estímulos empleados en todas las sesiones, tanto en las de entrenamiento como las de prueba, fueron blanco, rojo, verde y amarillo. El arreglo y el tipo de estímulos utilizados en cada sesión fue el mismo para los seis grupos. Durante las sesiones de entrenamiento y prueba se utilizaron como figuras círculos, triángulos, cuadrados y rectángulos; mientras que las sesiones de transferencia aparecieron como estímulos cruces, rombos, pentágonos y pares de líneas verticales, en los cuatro colores ya mencionados.

\section{Procedimiento}

Al inicio del estudio, a cada participante se le invitó a pasar a uno de los cubículos experimentales; se le pidió que se sentara frente al monitor y oralmente se le indicó que se trataba de un estudio sobre aprendizaje, agradeciéndoles su participación en el mismo. Al empezar cada sesión, se le pedía que leyera la descripción precontacto que aparecía en la pantalla y que dijera en voz alta lo que había entendido; si el reporte era adecuado respecto de lo leído se le pedía que continuara con la tarea, en caso contrario se pedía que volviera a leer hasta que el reporte fuera adecuado.

Durante todas las sesiones de prueba, Fase 1 y transferencia, se presentó la siguiente descripción precontacto de tipo ausente (A):

Bienvenido a la sesión

Te agradecemos tu participación

Durante la Fase 2, cada grupo recibió una descripción precontacto diferente, que difirió en la manipulación del criterio de ajuste de la tarea en el componente $R$, que se señala al lector en cursivas, aunque esto no aparecía en la pantalla al participante; las instrucciones fueron las siguientes:

-Específica Pertinente (EP):

Bienvenido a la sesión. Te agradecemos tu participación 
Tu tarea consiste en elegir, de entre aquellas que pueden ser seleccionadas, la figura que tenga el mismo color o forma (pero no ambas características al mismo tiempo).

Para llevar a cabo tu elección deberás de mover y oprimir el botón izquierdo del "mouse".

-Específica No Pertinente (ENP):

Bienvenido a la sesión. Te agradecemos tu participación.

Tu tarea consiste en elegir, de entre aquellas que pueden ser seleccionadas, la figura que tenga el mismo color y forma.

Para llevar a cabo tu elección deberás de mover y oprimir el botón izquierdo del "mouse"

-Genérica Pertinente (GP):

Bienvenido a la sesión. Te agradecemos tu participación

Tu tarea consiste en elegir, de entre aquellas que pueden ser seleccionadas, la figura que creas que tiene alguna relación.

Para llevar a cabo tu elección deberás de mover y oprimir el botón izquierdo del "mouse"

-Genérica No Pertinente (GNP):

Bienvenido a la sesión. Te agradecemos tu participación

Tu tarea consiste en elegir, de entre aquellas que pueden ser seleccionadas, la figura que tenga las mismas características.

Para llevar a cabo tu elección deberás de mover y oprimir el botón izquierdo del "mouse".

-Irrelevante (I):

Bienvenido a la sesión. Te agradecemos tu participación

Mientras respondes debes estar tranquilo y sentarte derecho en tu silla.

-Ausente (A):

Bienvenido a la sesión. Te agradecemos tu participación

Cada uno de los grupos se dividieron en dos subgrupos $(n=3)$; uno de ellos recibía retroalimentación continua, mientras que el otro recibió retroalimentación acumulada. En el caso de la retroalimentación continua, una vez elegido el estímulo se le daba al sujeto información sobre su ejecución con la aparición durante $1 \mathrm{~s}$, en la parte central de la pantalla, de un letrero de ¡ACIERTO! o ¡ERROR! de acuerdo con la selección realizada. Inmediatamente después aparecía el siguiente 
arreglo de estímulos, hasta completar los 36 ensayos de la sesión. No se programó límite de tiempo para responder, por lo que cada uno de los ensayos finalizaba hasta que el sujeto realizaba su elección.

Para aquellos participantes que recibieron retroalimentación acumulada, una vez que terminaba de realizar los 36 ensayos de la sesión, en la parte central de la pantalla aparecía un letrero que indicaba el número total de aciertos que había obtenido durante dicha sesión.

Al finalizar cada sesión, de prueba o experimental, a cada sujeto le aparecía en la pantalla un cuadro de diálogo en donde se le pedía que escribieran lo que acababan de hacer en la tarea (i.e. Describe con tus palabras la tarea que acabas de resolver), sin especificar qué era lo que debía redactar ni la finalidad de la descripción. Cuando el sujeto terminaba de redactar esta descripción poscontacto, se le pedía que abandonara el cubículo mientras se le programaba la siguiente sesión y se repetía el procedimiento ya descrito. Para cada sujeto, todas las sesiones experimentales y de prueba se realizaron en el mismo día.

\section{Análisis de Descripciones}

Para analizar las descripciones realizadas por los participantes, se utilizó la taxonomía propuesta por Ortiz, González \& Rosas (2008) que consta de seis categorías, que a continuación se presentan:

1. Descripción específica y pertinente (EP): aquella que menciona TODOS los elementos relevantes del componente en cuestión y cuya descripción de los mismos corresponde cabalmente con los elementos que estructuran la situación enfrentada.

2. Descripción genérica y pertinente (GP): aquella que sólo menciona alguno de los elementos relevantes del componente en cuestión, o que bien que excluye la mención de alguno de los elementos relevantes y cuya descripción de los elementos considerados corresponde cabalmente con los elementos que estructuran la situación enfrentada.

3. Descripción genérica y no pertinente (GNP): aquella que solo menciona alguno de los elementos adecuados del componente en cuestión, o bien que excluye la mención de alguno de los elementos relevantes, pero cuya descripción de los elementos contemplados no corresponda con los elementos que estructuran la situación enfrentada.

4. Descripción específica y no pertinente (ENP): aquella que menciona TODOS los elementos adecuados del componente en cuestión, pero cuya descripción de los mismos (por lo menos uno de ellos) no corresponde con los elementos que estructuran la situación enfrentada.

5. Descripción irrelevante (I): aquella que únicamente menciona elementos del componente en cuestión que NO se encuentran directamente relacionados con los requisitos del arreglo contingencial que permitirán al sujeto ajustarse a la situación, motivo por el cual resulta innecesario distinguir si es "específico o genérico" ni, más aun, "pertinente o no pertinente". 
6. Descripción ausente (A): aquella que no menciona NINGUNO de los elementos (pertinentes o no pertinentes) del componente en cuestión.

\section{Diseño}

Los sujetos fueron asignados aleatoriamente a uno de seis grupos experimentales $(n=6)$; todos lo sujetos enfrentaron individualmente un entrenamiento al "mouse", una prueba diagnóstica, dos fases experimentales (cuatro sesiones para cada una de ellas), una prueba, colocada entre las dos fases, una posprueba y una prueba de transferencia (ver Tabla 1). Con la finalidad de exponerlos a una historia experimental común, todos los grupos recibieron el mismo entrenamiento durante la Fase 1 manteniendo los componentes ausentes en la descripción precontacto pero dividiendo los grupos en dos tipos de retroalimentación (continua y acumulada).

En la segunda fase experimental, se manipuló la precisión de las descripciones precontacto (i.e. EP, GP, ENP, GNP, I o A) manteniendo la frecuencia de retroalimentación presentada durante la Fase 1.

Tabla 1.

Diseño y condiciones experimentales a las que se sometieron los sujetos en los distintos grupos.

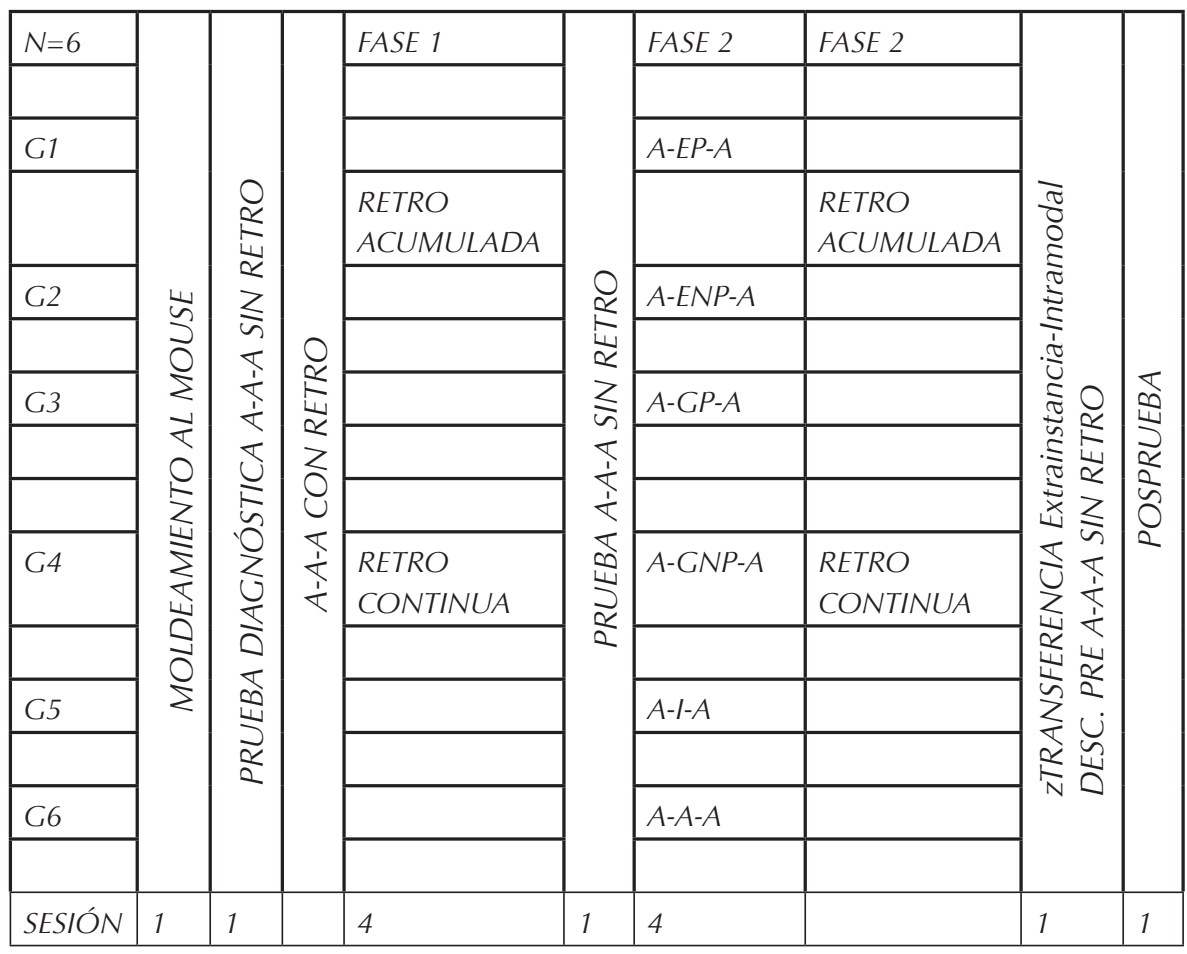



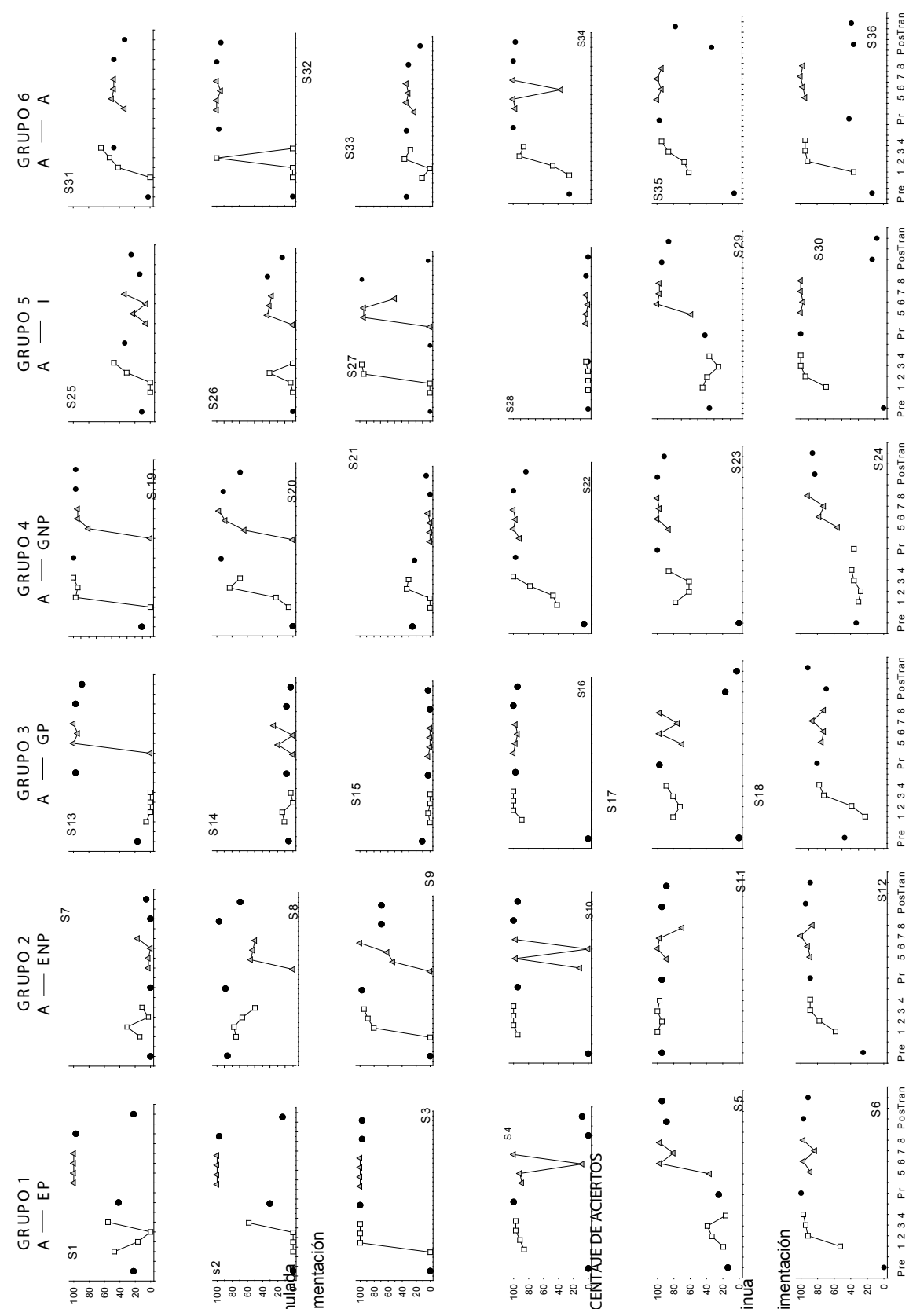

Figura 1. Porcentaje de respuestas correctas por sujeto en los seis grupos experimentales en relación con las dos frecuencias de retroalimentación. 
Resultados

En la Figura 1 se muestra el total de aciertos obtenidos por todos los participantes en cada una de las sesiones experimentales, de prueba y transferencia. En la parte superior se encuentran los participantes que recibieron retroalimentación acumulada y en la parte inferior se muestran los que recibieron retroalimentación continua a lo largo del estudio; al mismo tiempo, el tipo de descripción precontacto que recibieron se ve expresada en cada una de las columnas, desde la extrema izquierda (i.e. descripción de tipo EP) hasta la extrema derecha (i.e. descripción de tipo A). Los círculos negros muestran la ejecución en las pruebas y transferencia, mientras que los cuadrados blancos muestran el porcentaje de aciertos obtenidos en la Fase 1 y los triángulos blancos el obtenido durante las cuatro sesiones de la Fase 2.

En general, en la primera fase los porcentajes de respuestas correctas variaron de acuerdo con el tipo de retroalimentación que se les otorgó; así, 7 de los 18 sujetos que recibieron retroalimentación acumulada tuvieron una ejecución inferior al $40 \%$ de los aciertos, mientras que otros siete mostraron una mejoría conforme transcurrieron las sesiones (s3, s9, s19, s20, s27, s29 y s31). En esta misma Fase 1 , cuatro de los sujetos que recibieron retroalimentación continua (s4, s10, s11 y s16) tuvieron ejecuciones cercanas al 100\%, 9 sujetos iniciaron con niveles bajos de ejecución y mostraron una mejoría a lo largo de esta fase, al tiempo que los tres restantes mostraron ejecuciones por debajo del $40 \%$.

Durante la Fase 2, un total de seis sujetos tuvieron ejecuciones cercanas al $100 \%$, tres de los cuales pertenecían al Grupo 1 EP con retroalimentación acumulada (s1, s2 y s3). Tres sujetos, pertenecientes a los grupos con descripción precontacto de tipo GP (s15), GNP (s21) e I (s28), tuvieron ejecuciones de cero aciertos, dos de ellos recibieron retroalimentación acumulada (s15 y s21).

Tomando en cuenta el total de los participantes, seis mejoraron progresivamente su ejecución; tres de ellos recibieron descripciones precontacto de tipo Genérica No Pertinente (Grupo 4, s19, s20 y s24) y el resto formaron parte de los Grupos 2 (ENP, s8), 3 (GP, s13) y 5 (I, s29).

De acuerdo con los resultados obtenidos en las sesiones de Prueba, se muestra que en durante la pre y la posprueba 17 de los 36 participantes mejoraron su ejecución, al iniciar con niveles por debajo del $20 \%$ e incrementando a niveles de aciertos cercanos al $100 \%$ (i.e. s2 y s5), al tiempo que 12 de los participante mantuvieron el mismo porcentaje en la pre y posprueba. Con respecto a la prueba de transferencia, 18 participantes mostraron un porcentaje superior del $80 \%$; ocho de ellos (i.e. s13, s16, s18, s19, s20, s22, s23, s24) pertenecían a los grupos que recibieron descripción precontacto Genérica.

En la Figura 2 se muestra la distribución por tipo de descripción en cada componente para cada uno de los grupos en las dos fases experimentales (Fase 1 de lado derecho, Fase 2 de lado izquierdo). Cada grupo está subdivido en dos columnas, la 

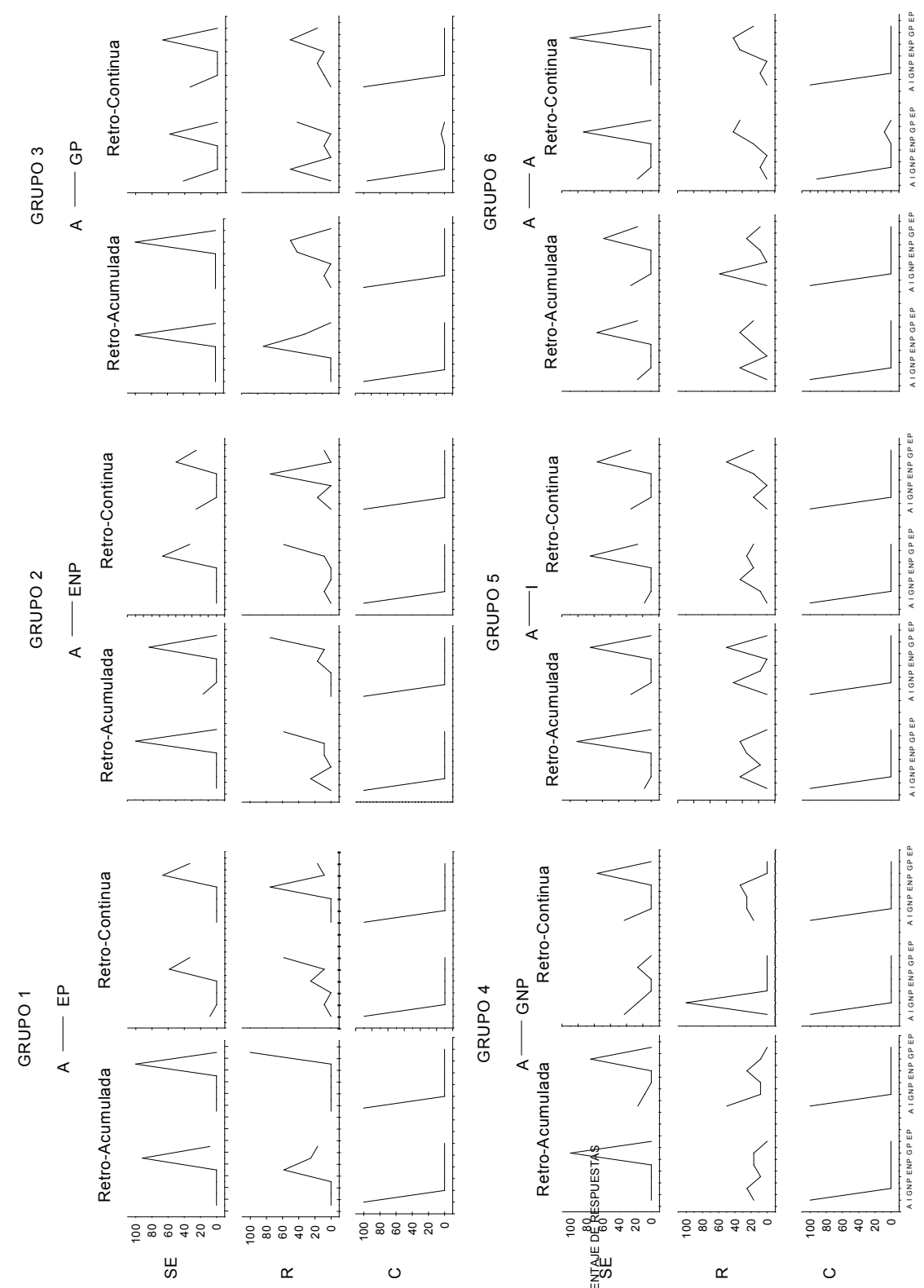

Figura 2. Porcentaje por tipo de descripción para cada uno de los componentes de la descripción poscontacto realizada por cada grupo durante todo el estudio. 
de lado izquierdo pertenece al grupo que recibió una retroalimentación acumulada y el de la derecha a los que recibieron una retroalimentación continua.

Se puede observar que el componente Consecuencia (C) se mantiene ausente en casi todas las descripciones de los sujetos sin importar al grupo al que pertenecen. Las únicas muestras de descripciones poscontacto distintas en este componente (e.g. Genéricas Pertinentes, GP) se encuentran en la Fase 1 de los Grupos 3 y 6 con retroalimentación continua.

El componente situación de estímulo (SE) fue descrito en su gran mayoría como GP, excepto en la Fase 1 del Grupo 4 con retroalimentación continua y algunas muestras de descripciones de tipo Ausente (A) en los Grupos 1 y 3 con retroalimentación continua, así como los grupos 5 y 6 con ambas frecuencias de retroalimentación. En la Fase 2, las muestras de descripciones de tipo Ausente se presentaron para los sujetos de los Grupos 2, 4 y 5 con ambas frecuencias de retroalimentación, así como en los grupos 3 continua y 6 acumulada.

Respecto del tipo de descripción relacionada con el componente Respuesta (R), los Grupos 1 y 3 con retroalimentación acumulada, mostraron una mejoría en cuanto al tipo de descripción poscontacto realizada al cambiar de la Fase $1 \mathrm{a}$ la Fase 2 (i.e. ausente a específica pertinente). Los grupos que mantuvieron descripciones poscontacto similares entre ambas fases del estudio fueron el Grupo 2, 5 y 6 con retroalimentación acumulada y el Grupo 4 con retroalimentación continua. Por otro lado, los grupos que empeoraron en sus descripciones poscontacto fueron los grupos 1, 2 y 6 con retroalimentación continua y el grupo 4 con retroalimentación continua (i.e. Específica Pertinente a Específica No Pertinente).

Los sujetos que tuvieron mejores ejecuciones instrumentales en la Fase 2 son los del Grupo 1 (cercanas al 100\% de aciertos), con retroalimentación acumulada, también realizaron descripciones de tipo Específica Pertinente en el componente Respuesta; los demás grupos muestran mayor variabilidad en el tipo de descripción realizada para el componente Respuesta.

\section{Discusión}

En general, los resultados muestran que los sujetos que recibieron retroalimentación continua en la Fase 1, mostraron mejores ejecuciones instrumentales que los sujetos que recibieron retroalimentación acumulada. Respecto a la Fase 2, se observan varios sujetos que mejoraron progresivamente su ejecución, cuatro de los cuales recibieron una descripción precontacto no-pertinente (GNP-ENP); asimismo, de manera global, el Grupo 1 (EP con retroalimentación acumulada), realizó las mejores ejecuciones y descripciones postcontacto.

En este estudio se observa que otorgarle al sujeto retroalimentación continua en conjunto con una descripción EP, parece no necesariamente proporcionar los elementos suficientes para realizar una buena ejecución pues si bien se podría esperar una ejecución instrumental muy alta durante todas y cada una de las se- 
siones, éste no fue el caso; de hecho, los resultados fueron superiores en aquellos sujetos que recibieron esta descripción precontacto de tipo EP pero con retroalimentación acumulada. Este dato, que parece estar contra la lógica de que a mayor información mejor ejecución, sin embargo, ha sido reportado en otros estudios (e.g. Guerrero-Radillo \& Ortiz, 2007; Ortiz et al., 2006; Ribes \& Martínez, 1990). Martínez \& Ribes (1996) sugieren que este efecto posiblemente se deba a que la retroalimentación continua no proporciona el tiempo suficiente para que se logre la abstracción de los elementos pertinentes de la tarea experimental, aspecto que se considera fundamental en el caso de la regla (cfr. Ribes 2000), lo que si sucede con la retroalimentación acumulada, "obligando" a que los sujetos se fijen a la retroalimentación, pasando por alto las características de los elementos que conforman la tarea así como de las relaciones pertinentes al criterio de respuesta de la misma.

Por otro lado, el hecho de que varios sujetos de los grupos en los cuales las descripciones precontacto no eran pertinentes (GNP con ambas frecuencias de retroalimentación) hayan mostrado una mejoría constante a lo largo de la segunda fase, puede deberse a que la descripción precontacto que adquirió una función instruccional durante la Fase 1 (en tanto el seguimiento de los sujetos y la consiguiente mala ejecución) dejó de funcionar como instrucción; debido a la manipulación del componente respuesta, la no pertinencia del criterio de ajuste a la tarea parece ser el factor responsable del abandono del llamado control instruccional. Estos datos concuerdan con lo propuesto por diversos autores en relación con la posible pérdida del control instruccional si las instrucciones (i.e. descripciones precontacto) no corresponden con las consecuencias durante un período extendido o si la operación no es pertinente para resolver la tarea (e.g. Baron y Galizio, 1983; Buskist \& Miller, 1986; Cerutti, 1994; Galizio, 1979; Hojo, 2002; Martínez \& Ribes, 1996; Martínez \& Tamayo, 2005; Ortiz, de la Rosa, Padilla, Pulido \& Vélez, 2008; Ortiz, Pacheco, Bañuelos \& Plasencia, 2007; Podlesnik \& Chase, 2006; Weiner, 1979).

Con respecto a la realización de las descripciones poscontacto, Guerrero-RadiIlo \& Ortiz (2007) realizaron un estudio con el objetivo de observar los efectos de la frecuencia de la retroalimentación y la precisión de la descripción precontacto sobre la ejecución instrumental y las descripciones poscontacto. Manipularon dos frecuencias de retroalimentación (i.e. continua e intermitente) y utilizaron descripciones precontacto ausentes y genéricas. Los resultados mostraron que la mayoría de las descripciones poscontacto realizadas por los sujetos, son de tipo GP en el componente situación de estímulo y de tipo A en el componente Consecuencia; mientras que en el componente $\mathrm{R}$ se muestra mayor variabilidad con casos de descripciones que pueden ser calificadas como de tipo EP, GP y ENP. Resultados parecidos se encuentran en el estudio de Ortiz et al. (2006) y Ortiz (2010) quienes sugirieron que la manipulación del componente R puede afectar en las descripciones poscontacto. 
En este sentido, es posible que los elementos explícitos en las descripciones precontacto, particularmente aquellos que integran el componente Respuesta, su precisión y la frecuencia de retroalimentación recibida permitan al sujeto abstraer los elementos necesarios para enfrentar la tarea, facilitando por un lado su ejecución instrumental y, por otro, la elaboración de descripciones poscontacto. Esto se puede ver reflejado por tres hechos: a) las mejores ejecuciones instrumentales se encontraron relacionadas con una mayor precisión en el componente $\mathrm{R}$ de las descripciones precontacto recibidas, b) en su mayoría, las mejores descripciones poscontacto realizadas por los sujetos fueron aquellas que eran seguidas tanto de descripciones precontacto más específicas como por mejores ejecuciones instrumentales y, c) dentro de este conjunto de descripciones poscontacto con mayor precisión, fueron aún mejores las descripciones poscontacto realizadas por sujetos que recibieron retroalimentación acumulada.

Sin embargo, la relación entre descripciones precontacto, ejecución y descripciones poscontacto no debe ser considerada directa, pues se encuentra modulada, entre otras cosas, por variables tales como la frecuencia, el tipo y modalidad de la retroalimentación (e.g. Ortiz, 2010; Ortiz \& González, 2010a; Ortiz, González, Rosas y Alcaraz, 2006; Guerrero-Radillo \& Ortiz, 2007), el tipo de tarea utilizada (e.g. Ribes, Moreno y Martínez, 2005) y el repertorio lingüístico con que cuenta el sujeto que facilite o no la identificación de los elementos pertinentes a la solución de la tarea (e.g. Ribes, 2000; Guerrero-Radillo y Ortiz, 2009).

De la misma forma, la precisión y pertinencia de cada uno de los componentes de la descripción precontacto puede, al menos lógicamente, contribuir en el efecto que tenga sobre la conducta del individuo. Ortiz \& González (2010a) realizaron un estudio con el fin de explorar los efectos de la manipulación del componente situación de estímulo (SE) en la ejecución instrumental y la elaboración de descripciones poscontacto en una tarea de igualación de la muestra de primer orden. Los participantes (estudiantes universitarios) fueron asignados a uno de los nueve grupos experimentales que se diferenciaron por el tipo de descripción precontacto recibida (i.e. tres diferentes tipos de precisión del componente $\mathrm{SE}$, específica pertinente y no pertinente, genérica no pertinente) y la frecuencia de retroalimentación recibidas (i.e. continua, acumulada o sin retroalimentación). De manera global, encontraron efectos diferenciales dependiendo de la precisión del componente SE y la frecuencia de retroalimentación que recibieron los participantes. Así, por ejemplo, cuando se utilizaron descripciones precontacto de tipo no pertinente (específica o genérica) en combinación con retroalimentación continua, los participantes inician cometiendo errores relacionados con la falta de correspondencia entre la descripción precontacto y las consecuencias recibidas (seguimiento instruccional), abandonando tal conducta instruida y apareciendo el control por las contingencias programadas. De igual forma, los resultados obtenidos por el grupo que recibió una descripción precontacto de tipo específica y no pertinente (ENP) en conjunto con retroalimentación continua, fue el que obtuvo 
ejecuciones instrumentales más altas y descripciones poscontacto más precisas y consistentes, tanto en sesiones de prueba, entrenamiento y transferencia. Este dato parece relevante pues sugiere la confirmación de la relación entre el grado de informatividad situacional que es otorgado tanto por la frecuencia de retroalimentación como por la precisión y pertinencia de la descripción precontacto.

El presente estudio partió del supuesto planteado por Ortiz y colaboradores (2006, 2008) de que el componente respuesta de las descripciones precontacto podría ser un factor crítico para la adquisición de una función instruccional por parte de dichas descripciones, supuesto que parece confirmarse con los datos aquí obtenidos. Sin embargo, para darle mayor robustez a esta sugerencia, es necesario seguir explorando el efecto de la manipulación de cada uno de los otros elementos que componen una descripción (i.e. situación de estímulo y consecuencia) así como las relaciones que se establecen entre dichos elementos y el componente respuesta.

\section{Referencias}

Baron, A. \& Galizio, M. (1983). Instructional control of human operant behavior. The Psychological Record, 33, 495-520.

Baron, A., Kaufmann, A. \& Stauber, K.A. (1969). Effects of instructions and reinforcement-feedback on human operant behavior maintained by fixed-interval reinforcement. Journal of the Experimental Analysis of Behavior, 12, 701-712. Available via: http://dx.doi.org/10.1901/jeab.1969.12-701

Buskist, W.F. \& Miller, H.L. (1986). Interaction between rule and contingencias in the control of human fixed-interval performance. The Psychological Record, $36,109-116$.

Catania, A. C., Shimoff, E., \& Matthews, B. A. (1989). An experimental analysis of rule-governed behavior. En: S.C. Hayes (Ed.). Rule-governed behavior: Cognition, contingencies, and instructional control. (Pp.119-150). USA: Plenum.

Cerutti, D.T. (1994). Compliance with instructions: Effects of randomness in scheduling and monitoring. The Psychological Record, 44, 259-269.

Chase, P. N. \& Danfort, J.S. (1991). The role of rules in concept learning. En: L.J. Hayes \& P.N. Chase (Eds.). Dialogues on Verbal Behavior. (Pp. 226- 235). Reno, NV: Context Press.

DeGrandpre, R. J., \& Buskist, W. E. (1991). Effects of accuracy of instructions on human. Behavior. The Psychological Record, 41, 371-384.

Galizio, M. (1979) Contingency-shaped and rule governed behavior: Instructional control of human loss avoidance. Journal of the Experimental Analysis of Behavior, 31, 53-70. Available via: http://dx.doi.org/10.1901/jeab.1979.31-53

Guerrero-Radillo, A. \& Ortiz, G. (2007). El papel de la retroalimentación y la ausencia o presencia de instrucciones en la elaboración de descripciones en tareas de discriminación condicional. Acta Colombiana de Psicología, 10, 5-13. 
Guerrero-Radillo, A.P. \& Ortiz, G. (2009). Efectos de un preentrenamiento verbal sobre descripciones poscontacto y ejecución instrumental. IPyE: Psicología y Educación. 3, 20-43.

Harzem, P., Lowe, C.F. \& Bagshaw, M. (1978). Verbal control in human operant behavior. The Psychological Record, 28, 405-423.

Hayes, S.C. (1989). Rule-governed behavior: Cognition, contingencies, and instructional correspondence control. New York: Plenum Press.

Hayes, S.C., Brownstein, A.J., Haas, J.R. \& Greenway, D.E. (1986). Instruction, multiple schedules, and extinction: Distinguishing rule-governed from Schedule controlled behavior. Journal of the Experimental Analysis of Behavior, 46, 137147. Available via: http://dx.doi.org/10.1901/jeab.1986.46-137

Hojo, R. (2002). Effects of instructional accuracy on a discrimination task. The Psychological Record, 52, 493-507.

LeFrancois, J. R., Chase, P. N., \& Joyce, J. H. (1988). The effects of a variety of instructions on human fixed interval performance. Journal of the Experimental Analysis of Behavior, 49, 383-393. Available via: http://dx.doi.org/10.1901/ jeab.1988.49-383

Martínez, H. \& Ribes, E. (1996). Interactions of Contingencies and instructional history on conditional discrimination. The Psychological Record, 46, 301-318.

Martínez, H., Ortiz, G. \& González, A. (2002). Precisión instruccional, retroalimentación y eficacia: Efectos sobre el entrenamiento y transferencia discriminación condicional en adultos. Acta Colombiana de Psicología, 8, 7-33.

Martínez, H., Ortiz, G. \& González, A. (2007). Efectos diferenciales de instrucciones y consecuencias en ejecuciones de discriminación condicional humana. Psicothema, 19, 14-22.

Martinez, H. \& Tamayo, R. (2005). Interactions of contingencies, instructional accuracy, and instructional history in conditional discrimination. The Psychological Record, 55, 633-646.

Newman, B., Buffington, D. M. \& Hemmes, N. S. (1995).The effects of schedules of reinforcement on instruction following. The Psychological Record, 45, 463476.

Ortiz, G. (2005). El papel de la retroalimentación y la precisión instruccional en la elaboración y uso de descripciones en tareas de discriminación condicional. Tesis Doctoral. Universidad de Guadalajara.

Ortiz, G., De la Rosa, E., Padilla, R., Pulido, E., \& Vélez, H. (2008). Efecto de la precisión e historia instruccional en la insensibilidad al cambio contingencial en tareas de igualación de la muestra de primer orden en humanos. Acta Comportamentalia, 16, 167-181.

Ortiz, G. \& González, V. (2010a). Efectos de Precisión y Pertinencia del componente Situación de Estímulo de una descripción precontacto. Revista Mexicana de Análisis de la Conducta, 36, 115-130.

Ortiz, G. \& González, V. (2010b). Efecto de dos tipos de descripciones precontac- 
to sobre la ejecución instrumental y descripciones poscontacto en tareas de igualación de la muestra. Acta Colombiana de Psicología, 13, 115-126.

Ortiz, G., González, A. \& Rosas, M. (2008). A taxonomy of the analysis of pre and post contingency contact descriptions. Acta Colombiana de Psicología, 11, 45-53

Ortiz, G., González, A., Rosas, M. \& Alcaraz, F. (2006). Efectos de la precisión instrucción al y la densidad de retroalimentación sobre el seguimiento, la elaboración y transmisión de descripciones en tareas de discriminación condicional. Acta Comportamentalia, 14, 103-130.

Ortiz, G., Pacheco, V., Bañuelos, I., \& Plascencia, L. (2007). Efecto del contacto con instrucciones, la especificidad e historia instruccional en la insensibilidad al cambio contingencial en tareas de igualación de la muestrade primer orden en humanos. Acta Colombiana de Psicología, 10, 107-115.

Peláez, M. \& Moreno, R. (1998). A taxonomy of rules and their correspondence to Rule-Governed behavior. Revista Mexicana de Análisis de la Conducta, 24, 197-214.

Podlesnik, A. C. \& Chase, P. N. (2006). Sensitivity and strength: effects of instructions on resistance to change. The Psychological Record, 56, 303-321.

Ribes, E. (2000) Instructions, Rules and Abstraction: A Misconstructed Relation. Behavior and Philosophy, 28, 41-55.

Ribes, E. \& Martínez, H. (1990). Interactions of contingencies and rule instructions in the performance of human subjects in conditional discrimination. The Psychological Record, 40, 565-586.

Ribes, E., Moreno, D. \& Martínez, C. (1995) Efecto de distintos criterios verbales de igualación en la adquisisción y transferencia de una discriminación condicional de segundo orden en humanos. Acta Comportamentalia, 3, 27-54

Ribes, E., Torres, C. \& Ramírez, L. (1996). Efecto de los modos de descripción en la adquisición y transferencia de una discriminación condicional en humanos adultos. Acta Comportamentalia, 4, 159-179.

Ribes, E. \& Rodríguez, M.E. (2001). Correspondence between interactions, performance, and self-descriptions in a conditional discrimination task: The effects of feedback and type of matching response. The Psychological Record, 51, 309-333.

Rosenfarb, I. S., Newland, M. C., Brannon, S. E., \& Howey, D. S. (1992). Effects of self-generated rules on the development of scheduled-controlled behavior. Journal of the Experimental Analysis of Behavior, 58, 107-121. Available via: http://dx.doi.org/10.1901/jeab.1992.58-107

Skinner, B.F. (1966). An operant analysis of problem solving. En: B. Kleinmuntz (Ed.). Problem Solving: Research, method and theory. (Pp. 225-257) New York: John Wiley and Sons.

Trigo, E. Martínez, R., \& Moreno, R. (1995). Rule performance and generalization in a matching-to-sample task. The Psychological Record, 45, 223-240.

Revista Mexicana de Análisis de la Conducta / Mexican Journal of Behavior Analysis v37 n1 4-2011/7-2011 
El papel de la precisión instruccional

Weiner, H. (1970). Instructional control of human operant responding during extinción following fixed-ratio conditioning. Journal of the Experimental Analysis of Behavior, 13, 391-394. Available via: http://dx.doi.org/10.1901/jeab.1970.13391 